\title{
Criminologie
}

\section{Les forces de l'ordre, expertes des climats urbains}

\section{Dominique Boullier et Stéphane Chevrier}

Volume 46, numéro 2, automne 2013

Nouveaux regards sur les métiers de la sécurité

URI : https://id.erudit.org/iderudit/1020990ar

DOI : https://doi.org/10.7202/1020990ar

Aller au sommaire du numéro

Éditeur(s)

Les Presses de l’Université de Montréal

ISSN

0316-0041 (imprimé)

1492-1367 (numérique)

Découvrir la revue

Citer cet article

Boullier, D. \& Chevrier, S. (2013). Les forces de l'ordre, expertes des climats urbains. Criminologie, 46(2), 131-148. https://doi.org/10.7202/1020990ar

\section{Résumé de l'article}

Les grands événements urbains nécessitent la production d'environnements sécurisés qui vont permettre de vivre le climat de la fête, de la manifestation ou du match (les terrains qui seront évoqués ici) dans un intérieur protégé. Les forces de l'ordre ont acquis une expertise dans le pilotage de ces climats, qui repose non pas sur des plans mais sur une acceptation d'être " à l'intérieur ", elles aussi, et non en surplomb. Elles mobilisent alors toutes les ressources du cadre bâti et le complètent par leur propre disposition pour créer des enveloppes, et elles utilisent aussi les ressources de leurs corps pour garder les sens en éveil pour une situation toujours susceptible de déborder. 


\title{
Les forces de l'ordre, expertes des climats urbains
}

\author{
Dominique Boullier ${ }^{1}$ \\ Professeur de sociologie \\ Sciences Po (Paris) \\ dominique.boullier@sciences-po.fr \\ Stéphane Chevrier \\ Sociologue \\ Bureau d'études Mana (études et conseil sociologiques) \\ ici.mana@orange.fr
}

RÉSUMÉ - Les grands événements urbains nécessitent la production d'environnements sécurisés qui vont permettre de vivre le climat de la fête, de la manifestation ou du match (les terrains qui seront évoqués ici) dans un intérieur protégé. Les forces de l'ordre ont acquis une expertise dans le pilotage de ces climats, qui repose non pas sur des plans mais sur une acceptation d'être "à l'intérieur», elles aussi, et non en surplomb. Elles mobilisent alors toutes les ressources du cadre bâti et le complètent par leur propre disposition pour créer des enveloppes, et elles utilisent aussi les ressources de leurs corps pour garder les sens en éveil pour une situation toujours susceptible de déborder.

MoTS-CLÉS • Maintien de l'ordre, ville, événement, sécurité, stratégie, intérieur.

\section{Introduction}

Les métropoles vivent au rythme des grands événements sportifs ou culturels et des manifestations (un match de foot, un festival de musique, une manifestation de rue, etc.) qu'elles coproduisent ou qu'elles accueillent dans les creux de leur morphologie (rues, places, parkings, terrains vagues, etc.) et dans des conteneurs de plus ou moins bonne qualité (stades, hippodromes, vélodromes, halls, etc.). La ville peut s'avérer plus

1. Dominique Boullier, Sciences Po, 27 rue Saint-Guillaume, 75337, Paris, FRANCE, Cedex 07 
ou moins accueillante pour ces manifestations, mais elle peut aussi se transformer, reconfigurer totalement l'espace existant pour proposer une nouvelle expérience aux participants déclarés comme aux tiers riverains qui sont aussi affectés. L'expérience de la ville en tant qu'espace de vie peut être radicalement transformée et les routines de ceux qui y vivent ou qui la gèrent, pour la sécurité comme pour toutes les autres activités, ne peuvent prétendre fonctionner à tous coups et doivent elles aussi être reconfigurées. Ces grands événements ponctuels ou plus réguliers, circulaires ou linéaires, diurnes ou nocturnes, participent à la définition de l'identité des villes et à la promotion de leur image dans le cadre de ce nouveau marketing territorial qui les met en concurrence. Ces grands événements, relayés par les médias, dépassent de loin leur territoire et leurs enjeux peuvent aussi largement dépasser la seule affluence à un concert. Dès lors la capacité à garantir l'ordre public devient une question cruciale, comme la sélection des villes pour les Jeux olympiques ou la Coupe du monde de football a pu le montrer. Pour comprendre les conditions de félicité des grands événements urbains, il convient de suivre l'activité de tous les êtres concernés, qu'ils soient professionnels ou amateurs, organisateurs ou publics, artistes ou forces de l'ordre, médias aussi bien que dispositifs matériels, comme le cadre bâti ou la barrière métallique. Tous ces points de vue sur l'événement concourent ensemble à le produire et aucun d'entre eux ne serait suffisant pour en rendre compte car aucun d'eux ne surplombe les autres.

\section{Une méthode multipoints de vue}

Toute méthode d'observation qui prétendrait rendre compte de ce que fait un événement à une ville ou de qui fait l'événement ne pourrait faire l'économie de ces points de vue divers ni celle des moments incertains où l'état des entités qui agissent n'est plus garanti (Latour, 2006) et où, comme on dit, tout peut basculer. Nous avons tenté d'analyser la fabrique de l'événement ${ }^{2}$ en suivant, à Paris, Nantes et Rennes, les publics et les professionnels des événements urbains, au cours de manifestations intersyndicales ou de mouvements identitaires (Marche des fiertés), de rassemblements festifs (raves), de festivals (Transmusicales)

2. Cet article est le fruit d'une recherche réalisée en 2008-2009 pour le Plan Urbanisme Construction Architecture du ministère de l'Écologie. Cette recherche a donné lieu à la publication d'un ouvrage: Boullier et al. (2012). 
et d'épreuves sportives (matchs de football). Notre méthode d'observation fut dénommée «méthode commando» (quatre chercheurs seniors et dix enquêteurs) car elle impose de multiples observateurs mobilisés au même moment sur les différents points de vue sur l'événement, sans pour autant prétendre reconstituer une impossible synthèse ou image globale mais plutôt les convergences, les conflits et les articulations (Strauss, 1992) entre points de vue. Nous souhaitons rendre compte ici seulement du suivi du travail des forces de l'ordre et des services de sécurité qui sont déjà particulièrement divers et dont l'articulation reste souvent problématique ${ }^{3}$. Il fallait que nous puissions être là au bon moment pour observer à la fois le travail de fabrication de l'événement et les compétences mobilisées pour le contenir. En effet, les professionnels de la sécurité développent de véritablement compétences pour sentir, lire, décoder, décrire les choses ou pour identifier des variations insensibles de l'ambiance et de ce que nous appellerons, à la suite de Sloterdijk (2005), le climat, comme le sens commun et les expressions courantes savent le faire d'ailleurs («c'est chaud, ce soir », «l'atmosphère était glaciale», etc.). Ces professionnels mobilisent des indices et cherchent des «appuis» (Dodier, 1993) pour qualifier les acteurs en présence et la «physionomie» de situations incertaines. Ils cherchent à saisir la dynamique des échanges pour mesurer les risques de débordement et de basculement qui peuvent conduire à l'affrontement et pour trouver le bon moment (kä̈ros) pour intervenir. La manière importe autant que le moment.

\section{Une expertise de l'intérieur}

Et au cœur de cette manière se trouve la nécessité d'être «à l'intérieur» (Boullier, 2011). La capacité à ressentir ce climat, cette ambiance, ses variations infimes et prémonitoires doit être incarnée, elle doit mobiliser le corps et ses sens, comme nous le montrerons dans notre dernière partie. Mais pour que cette opération soit encore possible, il est

3. Compagnies républicaines de sécurité (CRS), Brigades anti-criminalité (BAC), fonctionnaires de la Police judiciaire, policiers du Service d'Intervention et du Centre d'Information et de Commandement, motards de la police urbaine, gendarmes mobiles, Brigades motorisées de la gendarmerie nationale (BMO), Centre opérationnel de la gendarmerie (COG), Peloton de Surveillance et d'Intervention de la Gendarmerie (PSIG), agents de sécurité privés, services de sécurité des organisations syndicales, agents de sécurité, opérateurs de transport... 
indispensable que ces professionnels produisent des formes variées d'enveloppes qui permettent d'isoler le phénomène vis-à-vis des interactions complexes d'une ville dans son fonctionnement ordinaire. Ce travail est là encore un travail de production d'intérieurs. Certes, un centre de commandement pourra prétendre à la fois planifier et surplomber l'action pour la surveiller à distance. Ce léger mouvement de déplacement que nous appelons le niveau 1,5 (Latour et al., 2012), celui où les acteurs se donnent les ressources pour se voir agir, un peu, n'est pas négligeable, mais il reste dépendant des contacts, des capteurs et de tous les senseurs qui ont pu être installés au cœur même de l'événement. La compétence policière de maintien de l'ordre suppose l'acceptation d'être à l'intérieur, ce qui fait d'ailleurs tout le risque de ces métiers car se trouver pris à l'intérieur de cet événement peut engendrer des peurs, des réactions, et affecter tous les participants. La qualité des intérieurs produits à divers moments, par la disposition des forces, par les capteurs/agents de renseignement disposés aux bons endroits, par les tenues et équipements adaptés au climat et au moment, contribuera à orienter les événements vers des issues plus ou moins apaisées. La production de ces intérieurs, au sein desquels les forces de l'ordre elles-mêmes acceptent d'être, nécessite une expertise particulière qui nous a semblé peu étudiée ou sous-estimée. Il ne s'agit pas en effet seulement de catégorisations qui permettraient d'appliquer des consignes routinières en fonction de certaines populations (Viot, 2013), il ne s'agit pas non plus de se reposer sur les performances des technologies de surveillance en ajoutant caméra sur caméra, il faut apprendre à capter l'événement en son cœur, à le sentir vivre et donc à l'éprouver sans pour autant se mettre (trop) en danger. L'art de créer les bonnes distances sera l'une des ressources essentielles de cet exercice, ce qui suppose une compétence de spatialité (Lussault, 2007), non plus moderniste surplombante (Latour, 1992) mais bien éprouvée de l'intérieur. En ce sens, le travail du maintien de l'ordre est ancré dans la ville et dans les corps et constitue un travail profondément politique car comme le dit Arendt, «la politique prend naissance dans l'espace-qui-est-entre-les-hommes» (1995: 42). Climatiser, c'est bien rendre vivables ces intérieurs et le maintien de l'ordre constitue un des savoir-faire qui y contribuent. Leur compétence de «climatiseurs» sera le centre de notre analyse et, malgré ou grâce à son caractère réducteur, cette approche devra nous faire découvrir de nouvelles facettes de ces métiers, dans leurs dimensions d'expertise corporelle. 


\section{L'histoire d'une place en transe}

Pour rendre compte de ces intérieurs, rien ne vaut une narration, immersive, pour que le lecteur puisse éprouver lui aussi, en toute sécurité cependant, ce qui se passe au cœur même de ces événements. L'un de nos terrains d'enquête (les Transmusicales de Rennes) constituera notre point d'entrée. L'événement en question est appelé festival l'observation proposée ici porte sur une partie off présente chaque année -, et dont le caractère est incertain, ni programmé, ni interdit, ni véritablement équipé ou encouragé. Il serait possible de classer ces moments dans la tradition de l'analyse de la fête (et notamment des travaux qui ont aussi pris Rennes comme terrain, Beauparlant et al. [2006], Moreau \& Sauvage [2006]). Cependant, notre approche s'intéresse plutôt à la fois aux événements en général, à ce qui fait événement, et ici plus particulièrement au travail des forces de l'ordre et non à tous ceux qui contribuent à créer ces climats.

Les Transmusicales sont un festival d'hiver qui se déroule essentiellement au Parc des Expositions situé à la périphérie de Rennes (Bretagne). Des navettes de bus régulièrement cadencées établissent un cordon entre le centre-ville et le Parc des Expositions. L'espace principal du festival s'est petit à petit mis à distance de la ville, en raison notamment du succès important de ces concerts mais les organisateurs ont su aussi produire un intérieur très savamment climatisé par des moments et des lieux forts et des moments et des lieux de décompression. Mais parallèlement aux concerts organisés dans les différents halls $\mathrm{du}$ Parc, des musiciens se produisent dans les bars du centre historique de la ville. À la fermeture des bars, la fête off se poursuit, jusqu'à l'aube, dans les quelques places (Place Saint-Michel et Place Sainte-Anne) et rues adjacentes. Une rave party (Epstein \& Fontaine, 2006) est traditionnellement organisée dans le département par un collectif de sound systems en parallèle du festival. De nombreux attracteurs périphériques sont donc présents et il n'est pas simple de produire l'enveloppe qui contiendrait toutes ces émergences associées à l'événement. Le travail des forces de l'ordre en est rendu d'autant plus complexe et mobilise pendant trois jours et trois nuits d'importants moyens de police et de gendarmerie. 
Rennes (place Saint-Michel) - Vendredi 5 décembre 2 h 00 du matin

Les bars viennent de fermer leurs portes. Une foule joyeuse et enivrée se déverse dans les rues et s'installe sur l'une des petites places (place Saint-Michel) du quartier historique. Cette place est desservie par quatre rues dont l'une descend vers le canal qui traverse la ville. La place des Lices, voisine, accueille tous les samedis un grand marché. Depuis minuit, la lieutenante $\mathrm{C}$ - qui coordonne les effectifs de police - alterne les allers-retours entre son bureau à l'hôtel de police et la place, ou les rues encombrées dans lesquelles elle déambule. Elle est en tenue civile, mais porte son arme de service à la ceinture. Elle est au contact des «fêtards» qui parfois l'interpellent gentiment. La situation est «calme», selon la lieutenante C. Les policiers de la BAC (Brigade anti-criminalité) présents dans la foule, en tenue civile, remontent régulièrement des informations par talkie-walkie. Le centre reste ainsi bien connecté grâce à ces capteurs d'information et de sensation et peut se considérer encore à l'intérieur tout en étant à distance. Rien dans la «physionomie» de la situation ne met en éveil la lieutenante $\mathrm{C}$ :

Il faut avoir l'œil. Tout est calme, puis tout d'un coup, ils deviennent agressifs. On les voit s'enivrer, on sait qu'il ne faut pas grand-chose pour que ça parte. Au début, ils sont éméchés, c'est jovial, quand ils passent à côté de nous, ils veulent discuter, ils font des blagues, c'est pas agressif, c'est gentil. Après, ils s'alcoolisent un peu plus et nous lancent des regards méchants. On sait que ça monte. On les dérange dans leur alcoolisation. Ils vont avoir une insulte, un geste, une provocation...

Nos enquêteurs qui sont dans la foule notent (sur leurs carnets) que les «esprits s'échauffent». Si la situation est encore «bon enfant» (expression favorite en maintien de l'ordre), quelques fêtards se battent entre eux. Les patrouilles de la section d'intervention (policiers en tenue) constituées de trois fonctionnaires passent à six et restent désormais en périphérie des regroupements. L'enveloppement change de forme et le cadre bâti de la place ainsi que les personnels de police coopèrent pour le produire.

\section{2 h 15 Simon (central)}

Lieutenante $\mathrm{C}$ à Orion nuit: L'équipe Rivarol nous signale un fort feu de palette au niveau de la place Saint-Michel. Un feu de joie avec pas mal de monde autour. Ils demandent la conduite à suivre. 
Orion nuit: C'est reçu Simon. Pour l'instant, ils n'interviennent pas. Vous faites appel aux pompiers. On va rassembler les effectifs (BAC et SI, Service d'Intervention).

Nous sommes à ce moment dans une situation d'incertitude. L'importance du feu n'est pas connue. Il existe un fort risque d'incendie dans ce quartier constitué de maisons en bois. Toute allusion au feu à Rennes mobilise immédiatement une tradition, des récits, voire des mythes qui sont extrêmement sensibles chez les habitants comme dans les forces de police et les autorités locales (grand incendie de 1720, incendie du Parlement de 1994). On chante, on danse, on crie autour du feu, on tape sur les poubelles transformées en djembés. Il faut impérativement créer des attracteurs et ce que nous appelons un contenant, c'est-à-dire ce qui va mobiliser l'attention, dès lors que la nuit avance et qu'aucun programmateur n'est chargé de cette animation. Or, une foule sans attracteur ne peut devenir un public (Boullier, 2010), ce qui la rend nettement plus incertaine. Les esprits des participants racontent une histoire de feu de joie, ceux des forces de l'ordre rappellent une histoire de feu dramatique. La lieutenante $\mathrm{C}$ a la possibilité de faire venir en renfort des CRS stationnés à quelques centaines de mètres. Elle s'équipe d'un gilet pare-balles et d'un tonfa (matraque) pour aller au cœur de la foule et au plus près du feu. Le bruit est assourdissant (chants, rires, cris). Le feu est très modeste, mais c'est la présence de cracheurs de feu qui lui laisse craindre que la situation ne dégénère. La lieutenante n'observe pas de loin, elle pénètre dans la foule, elle veut être à l'intérieur pour être sûre d'avoir une captation fine d'informations, mais elle doit cependant protéger son intérieur à elle et s'équipe pour garder une distance moins risquée malgré la proximité.

$2 \mathrm{~h}$ 29. Le fourgon pompe tonne (FPT) des sapeurs-pompiers arrive sur le site. Quatre ou cinq policiers protègent le fourgon situé à une dizaine de mètres du feu. Une dizaine de CRS, casqués et matraque en main arrivent au pas de course. La foule recule, la tension est palpable: "C'est bon, ils sont arrivés. Yes, c'est bon ça. Alors ça va commencer», s'exclame un fêtard à la vue des CRS. Là encore, des attentes sont mobilisées qui font appel à des histoires partagées, celle des bagarres rituelles pendant cinq ans entre les forces de l'ordre et les étudiants tous les jeudis soirs sur une place voisine. La police risque dans ce cas de devenir l'attracteur, ce que craignent plus que tout les responsables. Ce 
fêtard, lui, semble apprécier qu'un véritable événement puisse se dérouler dans l'événement.

Les CRS sont tendus. Ils ne parviennent pas à maintenir un cordon de sécurité autour des pompiers. Les CRS n'ont pas leur tenue de maintien de l'ordre complète. Il leur manque leur bouclier et leurs protections corporelles. Ils portent leur casque, mais la visière n'est pas rabattue. La tension monte encore d'un cran lorsque les CRS mettent un masque en papier pour se protéger des inhalations de gaz lacrymogènes. Ce détail n'a pas échappé aux fêtards qui l'interprètent comme une velléité d'en découdre.

Les signes extérieurs (équipement des CRS) suffisent à transmettre une information et à produire une interprétation partagée de la situation. «Monitorer le climat» suppose ainsi d'agir sur les images et sur les attentes, ce qui vaut dans les deux sens, puisque les fêtards savent aussi en jouer pour faire réagir les forces de l'ordre et les «chauffer». Les situations peuvent ainsi dégénérer largement en raison d'interprétations erronées ou anticipées de signes donnés par les uns ou les autres.

Le feu est éteint. Pour apaiser la situation, la lieutenante $\mathrm{C}$ demande aux CRS de se replier:

On nettoie le feu, on part vite et on les laisse finir leur soirée. Plus on est présent, plus ça va dégénérer. Tant que ce n'est pas l'heure, entre guillemets, de dire «maintenant tout le monde s'en va, la fête est finie», j'aime autant éviter que ça dégénère. C'est encore trop tôt (...). Notre présence sur place risque de réactiver l'événement. Ça va se diluer tout seul, sauf s'il y a un noyau dur qui décide de remettre le feu.

Si la situation s'est calmée, la lieutenante prévient le niveau supérieur de sa hiérarchie et retourne à l'hôtel de police pour s'équiper de sa tenue de maintien de l'ordre:

Si ça repart, je ne peux pas rester observer en civil. Je me mets en civil pour aller voir quand c'est calme, normalement, je suis censée être en tenue. Tant que c'est calme, je préfère y aller en civil, on peut se balader dans le dispositif, en tenue, on n'aurait pas pu se balader. Quand on sent que ça monte, on met la tenue de MO.

Tout le travail d'ajustement des tenues fait partie de l'expertise sur la gestion des distances, des indices qui créent des dispositions à agir. Ce sont les corps des forces de l'ordre et leurs équipements qui jouent ici ce rôle de cadrage de l'attention et qui sont, on le voit, manipulés avec précaution. 
Nous sommes sur le même lieu avec les mêmes acteurs. Mais la situation est totalement différente. Il est $4 \mathrm{~h} 00$ ce samedi matin. Les policiers estiment qu'ils peuvent mettre fin à la fête pour laisser place aux services d'entretien de la voirie et aux commerçants qui s'installent pour le marché du samedi. Les policiers (Service général et CRS) sont en tenue de maintien de l'ordre. Ils sont en nombre pour charger et bloquer les rues qui conduisent à la place. L'enjeu est l'évacuation de la place pour laisser place à d'autres usages. Mais la situation peut basculer:

La difficulté, c'est qu'ils sont dans un tel état d'ivresse qu'ils n'arrivent pas à comprendre ce qu'on leur dit. Avec 2,5 grammes en moyenne, vous pouvez leur expliquer ce que vous voulez... le risque, c'est qu'une foule qui n'est pas hostile le devienne lors de l'intervention, d'où les précautions pour ne pas faire prendre de risques au personnel. Je ne sais absolument pas comment ils vont réagir. A priori, on sent qu'il n'y a pas de tension. On est suffisamment nombreux pour ne pas avoir à employer la force. Dans ce type d'événement, quelquefois, nous avons affaire à des mouvements libertaires qui profitent de l'agglomération des gens pour faire dégénérer la situation. (Commandant A)

Leur seule présence permet de faire fuir une partie du public. L'annonce faite au mégaphone par le commandant A permet d'inviter les personnes à rentrer chez elles. Les policiers qui bloquaient les rues convergent progressivement et tranquillement vers le centre de la place. Mais à mesure que les publics se décantent, la tension monte. La BAC protège les arrières des policiers en tenue. Des insultes et des canettes de bière commencent à voler. Les fêtards cèdent du terrain et reculent vers le canal. Le profil de la rue en pente est favorable aux policiers qui poussent les fêtards. Lorsque les policiers estiment qu'ils ont trié les publics, que ne restent que les «plus durs», ils procèdent par bonds successifs déclenchés au sifflet pour repousser les derniers fêtards. La BAC interpelle quelques personnes. Les derniers fêtards sont repoussés de l'autre côté du canal. Les rues sont de nouveau ouvertes à la circulation.

Toute l'opération a duré trente minutes et se révèle être désormais routinière chaque année pour les forces de l'ordre, mais aussi pour les fêtards. La perception du risque de basculement dépend de l'expérience certes, mais aussi de la prise d'information qui demande d'être à l'intérieur, d'observer l'état des fêtards pour dire ce que l'on sent. Il est 
impossible de construire un dispositif de mesure du climat d'une telle situation et pourtant, les experts que sont les responsables des forces de l'ordre savent capter avec assez de précision ce climat pour proportionner leur riposte. Ici la place a joué un rôle essentiel pour faciliter le travail de décantation puisqu'il n'y avait qu'une seule issue ouverte, que la place des Lices adjacente est en longueur et en pente, et permet de faciliter l'écoulement. Ce sont bien des catégories physiques décrivant des corps, des pressions, des écoulements qui constituent le répertoire d'analyse à ce moment de l'intervention.

\section{Les ressources du cadre bâti étendu}

Les organisateurs, les services de sécurité et les forces de l'ordre ont cherché à contenir l'événement dans des conteneurs à l'accès strictement contrôlé (stades, parcs des expositions, salles de spectacles, usines désaffectées), dans lesquels ils disposeront de certaines prérogatives et de certains monopoles, et à l'encadrer en déployant des hommes et des moyens opérationnels autour de ces conteneurs. Lorsqu'ils ne peuvent s'appuyer sur ces ressources construites ad hoc, ils doivent «faire avec» les propriétés du cadre bâti et y trouver des alliés, des «amis», disent même les sapeurs-pompiers (Boullier \& Chevrier, 2000). Ils bénéficient ainsi dans le récit précédent de la forme de la place, de taille modeste et aisément close par la fermeture des accès. Leur propre action collective, en corps constitué pourrait-on dire, vient compléter cette enveloppe qu'ils créent et permet de circonscrire le risque. Ils doivent produire une forme d'immunité (Sloterdijk, 2005) vis-à-vis du reste de la ville, ce qui est exactement l'inverse de la communication. Cependant, ce n'est pas toujours aussi aisé. Car l'événement, pour faire événement, se doit de ne jamais être tout à fait prévisible, c'est même pour cette raison que le public souhaite "y être», «en être», «être là», alors qu'il pourrait écouter le disque ou regarder tout cela à la télévision, pour certains. L'événement reste instable et crée une forme permanente d'incertitude et de tension car il ne peut être réduit au programme imaginé par ses organisateurs ou ses initiateurs. Il menace sans cesse de déraper, d'être détourné, d'échapper à ses organisateurs et de déborder hors des enveloppes créées. Le vocabulaire (débordement ou dérapage) dit bien le risque de voir l'événement sortir du cadre et des limites dans lesquels on voudrait l'inscrire. Des événements parallèles plus ou moins spontanés peuvent se développer au cœur ou en marge 
de l'événement central, déplaçant ainsi son centre de gravité ou son foyer. Faire événement, c'est ainsi organiser l'économie des attentions (Goldhaber, 1997; Boullier, 2009) pour éviter une trop grande dispersion dans un espace public soumis à la concurrence des producteurs de micro-événements ou au contraire pour assurer grâce à une telle dispersion quaucun seuil critique ne sera franchi. Mais ce fractionnement en micro-événements peut aussi constituer autant de points d'échauffement qui, par contagion, de proche en proche, peuvent modifier le climat et affecter la ville tout entière. Dans chaque cas, les méthodes et les équipements mobilisés seront différents pour suivre, encadrer ou enfermer les participants selon les degrés de risque et de porosité qui paraissent supportables.

Lorsque l'événement se déroule dans une enceinte fermée, il est doublement environné, renforçant ainsi l'effet d'insularité. Il est contenu dans un conteneur de béton et de fer (un stade par exemple) qui permet d'accueillir, de trier, de filtrer, de canaliser, de contrôler, de ranger, d'évacuer des publics variés. Ce conteneur est lui-même ceinturé par un «cordon» de policiers ou de gendarmes lourdement équipés qui en contrôlent les points accès. Ces dispositifs humains et techniques d'encadrement permettent d'encercler (au sens premier du terme) et de contenir de manière toujours provisoire l'événement dans un périmètre aux contours bien définis. Dans un contexte beaucoup moins enveloppant, tous les ronds-points à proximité du Parc des Expositions qui accueillent le festival des Transmusicales (Rennes) sont tenus par les forces de l'ordre qui procèdent à des contrôles systématiques d'alcoolémie. Toutes les rues qui permettent d'atteindre la place Saint-Michel sont équipées de barrières et interdites à la circulation les jours de festival. Les forces de maintien de l'ordre sont stationnées à bonne distance. De même, tous les accès routiers menant à la rave qui s'est tenue en marge de ce festival en 2007 sont contrôlés par les forces de l'ordre qui procèdent là encore à des contrôles d'alcoolémie et parfois de stupéfiants. La manifestation (autorisée) des teufeurs (ravers) qui s'est déroulée dans les rues de Rennes en 2008 pour protester contre l'interdiction de se rassembler est discrètement encadrée par un dispositif policier qui progresse «en tiroir» et ouvre et ferme la manifestation. Les forces de l'ordre dessinent une barrière qui isole et imperméabilise l'événement pour produire un lagon contrôlé au sein de l'océan urbain. Si l'imperméabilité est parfois très relative, elle renforce cependant le caractère quasi insulaire du lieu de l'événement. Par ailleurs, cet envi- 
ronnement est ceinturé par une troisième couronne au profil très différent, car apparemment immatérielle, que nous n'évoquerons pas ici et qui se déroule aussi dans le spectre des radiofréquences.

Toute l'histoire que nous avons racontée montre bien que les décisions ne sont pas seulement appliquées du haut en bas, elles proviennent du terrain, sont proposées depuis le terrain (car des officiers sont sur place) et sont mises en œuvre dans des formes particulières sur le terrain (Mann, 1994). Le modèle du plan a été déjà largement critiqué depuis les travaux de Suchman (1987) notamment, l'auteure indique que le plan n'est pas ignoré mais qu'il est mobilisé comme une ressource parmi d'autres, comme le font les kayakistes qui utilisent leur guide pour anticiper la vision de la rivière avant de la descendre tout en sachant très bien qu'il n'y aura aucune application stricte de procédure standard. De la même façon, les méthodes des bonds que l'on voit mises en œuvre pour vider la place sont à l'évidence des scripts déjà répétés (Latour, 2012). Mais ce script est seulement une ressource qui n'est pas «appliquée» mais qui permet de connecter l'action avec le terrain avec un équipement plus riche. L'engagement «à l'intérieur» oblige à reformuler tous les scripts.

\section{Un monde à portée de main: les équipements du corps-à-corps}

Le cadre bâti ne permet pas à lui seul de créer les enveloppes nécessaires. Il faut en effet le compléter, l'adapter avec d'autres équipements comme les barrières mais aussi avec les corps des forces de l'ordre. Le choix judicieux de l'équipement fait partie de l'expertise du maintien de l'ordre (Bruneteaux, 1996), car il faut assurer à la fois le contact et la communication (on reste à l'intérieur) tout en garantissant une certaine immunité (une protection). Tout système immunitaire n'est d'ailleurs jamais un système étanche, mais un régulateur des échanges. L'idéal de l'extériorité et de l'immunité serait alors de piloter à distance des machines pour contrôler un événement qui n'affecterait en rien ceux qui le surveilleraient («comme le lait sur le feu», dit-on couramment dans la police) : c'est la politique du drone (Chamayou, 2013). Nous montrons ici, contre ce modèle moderne par excellence du surplomb, à quel point piloter un climat, le climat d'un événement, nécessite une présence à l'intérieur pour laquelle les corps des intervenants restent les capteurs indispensables. Les corps sont cependant équipés de façon très 
variée. Les jambières, les gants, le gilet pare-balles, le bouclier, le casque, la matraque, l'arme de poing portée à la ceinture, etc., disent bien la possibilité de voir l'événement se transformer en un affrontement à distance ou un corps-à-corps. Toute la question des bonnes distances est ainsi documentée (Garfinkel, 1967) pour les chercheurs, mais aussi pour les participants. Le bouclier et la matraque, presque anachroniques, renvoient à la forme archaïque et traditionnelle du combat. Ces outils rappellent que le combat consiste en premier lieu à toucher le corps de l'autre et à se protéger de ses assauts. En milieu ouvert, le sentiment d'encerclement provoqué par une proximité trop forte ou le port de ces outils peut être perçu comme une provocation et une invitation à l'affrontement et au corps-à-corps avec les forces de l'ordre. Les officiers de police et de gendarmerie en sont conscients et maintiennent leurs hommes à bonne distance. Ainsi, les Compagnies républicaines de sécurité qui encadrent les fêtes de rue en marge des Transmusicales (Rennes) sont placées à l'abri des regards et en retrait de l'épicentre de l'événement. Cette mise à distance nécessaire ouvre donc un espace.

Le port d'un masque à gaz à la ceinture nous rappelle que le combat peut être d'une tout autre nature. Les forces de l'ordre peuvent projeter des gaz lacrymogènes par bombe aérosol individuelle ou par jet de grenades. La possibilité d'utiliser des gaz souligne une évolution du rapport de force. Il ne s'agit plus ici de toucher le corps de ses adversaires mais de modifier et de maittriser leur environnement. Sloterdijk (2005) nous rappelle que l'homme est fondamentalement un «êtredans-l'air». En projetant des gaz, les forces de l'ordre privent leurs adversaires d'un environnement et d'un milieu nécessaires à la vie et exploitent à leur avantage cet intérieur qui nous est commun, l'atmosphère. À l'inverse, en portant un masque à gaz, elles s'affranchissent d'un air qu'elles ont modifié pour le rendre irrespirable. Elles se ferment et s'isolent du milieu extérieur dans une installation climatique, dans un corps-capsule, dans une tenue-prothèse qui tient lieu d'environnement. Ce n'est qu'au prix d'une fermeture et d'une introversion qu'elles peuvent évoluer de manière autonome dans ce milieu. Aller trop loin dans cette direction, c'est cependant prendre le risque de perdre tout contact sensoriel avec ce milieu et de ne pas mesurer des changements pourtant décisifs.

La mise en scène d'un possible emploi des gaz permet ainsi une gradation de la force qui est clairement perçue par les participants et provoque un tri parmi eux. Sur la place Saint-Michel à Rennes, de 
jeunes fêtards ont vu quelques CRS s'équiper d'un masque en papier. Avant même son emploi, le gaz a un effet dispersant, car l'équipement «masque» documente à lui seul un répertoire d'actions déjà connu, qui permet d'anticiper la suite des événements: «ça va cogner ou ça va gazer». Cette trace mémorielle d'un apprentissage collectif est une ressource importante car elle permet d'économiser l'emploi effectif de la force pour seulement en afficher les signes.

\section{Un oasis de sons}

Agir sur le climat, sur le cosmos, sur ce qui nous entoure et dont on ne peut se détacher peut se faire en affectant l'air que l'on respire. Mais cela peut se faire aussi à travers le son, matériau invisible, vibration qui se propage et dont il est difficile de se protéger car les corps humains ne sont pas équipés de paupières pour les oreilles! Nous sommes donc par définition à l'intérieur de ce que Sloterdijk (2005) appelle un «phonotope» et l'action sur cet intérieur est extrêmement efficace pour modifier le climat d'une fête, d'un événement quel qu'il soit. L'événement est aussi et en grande partie une expérience acoustique. Il ne peut y avoir d'événement sans bruits de pieds, sans claquements de mains, sans grondements, sans cris, sans chants, sans musique, sans roulements de tambour, sans insultes lancées à la face de son adversaire, etc. L'événement est un «oasis de sons» (Sloterdijk, 2005) et un paysage sonore qui isolent de l'extérieur. Les sons sont nécessaires à la construction du collectif et au dépassement des frontières proxémiques (Hall, 1984) que maintient la personne dans son rapport à l'autre. Les sons, et particulièrement les basses des sound systems des teufeurs, ne connaissent pas les frontières, ils pénètrent les corps et résonnent en chacun d'eux (Queudrus, 2001). Ainsi les bruits et les sons redondants ont d'abord une «visée chorale» (Gagnepain, 1994), ils ont pour but premier de rendre tangible et palpable le groupe qui se manifeste à lui-même sa propre existence (Champagne, 1984), de construire un être-ensemble. Si l'enjeu est de construire ce collectif, l'événement est constitué, le plus souvent, d'une multitude de «phonotopes» ou de foyers sonores qui ne sont jamais tout à fait sur la même longueur d'onde. Si l'architecture du conteneur (un stade par exemple) permet dans certains cas de «designer» les sons pour construire virtuellement un collectif, en milieu ouvert, à l'air libre, dans l'espace public, ces conditions ne sont pas toujours réunies. Le choix du lieu est donc primordial. Les rues canyons 
de telle préfecture offrent aux manifestants une caisse de résonance qu'il faudra bientôt abandonner à d'autres pour poursuivre le chemin. La place Saint-Michel, ni trop grande, ni trop petite, située à proximité des bars de nuit, constitue un lieu d'accueil parfait pour construire une bulle sonore au rythme des djembés ou des poubelles devenues percussions.

Les tambours des supporters de foot, les porte-voix et les sirènes des manifestants ou les murs de sons des teufeurs sont les indispensables outils du design sonore. Les coulisses de l'événement (bus, couloirs de métro, gares, places aux abords du stade) qui conduisent vers le point de rassemblement ont très souvent une fonction de lieu de répétition. On s'y chauffe la voix. L'action des forces de l'ordre repose pourtant sur ce découpage d'un espace sonore qui limite les nuisances tout en sachant qu'il est quasiment impossible en ville de produire cette immunité que l'on cherche pour séparer des publics aussi différents. L'enveloppe que se créent les fêtards devient plus forte une fois qu'elle est sonorisée car elle contamine tout le reste de la zone et affecte les riverains. Mais elle affecte aussi les forces de l'ordre qui doivent s'assurer de pouvoir communiquer, avec leur centre mais aussi entre elles et la perte de canal de communication sonore finit par saper toute la puissance d'action d'un corps professionnel (Dieu, 1993). C'est pourquoi le mégaphone (pour faire les sommations vis-à-vis des derniers fêtards), les sifflets (pour rythmer les bonds des CRS), les talkieswalkies et les téléphones portables sont des équipements indispensables pour garder une forme de maîtrise du climat sonore. L'importance de ce canal est souvent sous-estimée depuis que toutes les ressources sont affectées aux caméras et à la supposée toute-puissance de l'image. Les types d'attention mobilisés sont de nature différente selon les canaux employés et le caractère profondément corporel du son constitue une ressource remarquable pour l'expérience de l'événement de l'intérieur, aussi bien pour les publics que pour les forces de l'ordre.

\section{Conclusion}

Les professionnels des climats urbains ne peuvent guère prétendre contrôler totalement ces situations complexes, évolutives, incertaines. Ils ne peuvent guère adopter cette posture moderne que Sloterdijk (2005 : 9) caractérise par une supposée extériorité: «est moderne celui qui croit n'avoir jamais été à l'intérieur». Or, les forces de l'ordre comme les organisateurs et tous les participants sont à l'intérieur et le savent, 
malgré tous les espoirs mis dans la vidéosurveillance: leur expertise collective consistera précisément à créer les conditions d'un monde intérieur vivable, de leur point de vue. Pour cela, il faudra accepter d'être affecté, tout en mobilisant toutes les ressources pour ne pas se laisser capter. Il est aisé de comprendre alors à quel point l'expertise relève d'un art de faire où le corps conserve la trace de ces apprentissages et qui sera dès lors considéré comme tacite et non codifiable. En réalité, sans atteindre le formalisme des savoir-faire des pompiers, nous avons pu voir à l'œuvre une expertise de plus en plus déclarée, transmise et codifiée, dans un domaine pourtant aussi impalpable que le climat urbain. Cette dimension de l'expertise des forces de l'ordre (Chevrier et al., 2002), qui savent créer des intérieurs, des enveloppes, et qui dans le même temps, doivent accepter d'être à l'intérieur de ces enveloppes qu'elles ont créées, est rarement mise en avant. Il est plus aisé en effet de continuer à penser les dispositifs de pouvoir comme mis en œuvre par des acteurs-stratèges, pour qui toutes les ressources (les objets notamment mais aussi l'environnement ou l'expérience) ne font que suivre leurs consignes, les plans et les scripts. Mais les professionnels eux-mêmes nous ont montré à quel point ils acceptent de «devenir avec» (Haraway, 2003) l'événement et que leur capacité à jouer un rôle dans le pilotage de ce climat repose sur leur savoir-faire d'immersion dans des intérieurs risqués, mais en étant suffisamment équipés pour éviter le débordement total de l'enveloppe. Qui aurait dit que les forces de l'ordre nous démontreraient qu'elles mettent en œuvre des méthodes de non-modernes, celles de la seconde modernisation (Beck, 1992), qui exigent réflexivité et affectation?

\section{Références}

Arendt, H. (1995). Qúest-ce que la politique? Paris: Le Seuil.

Beauparlant, C., Darris, G., Lemoine, A., \& Léon, H. (2006). La ville, la nuit. Rennes et Nantes, de nouvelles exigences de gestion urbaine. Cabiers de la sécurité, 61 (2), 85-107.

Beck, U. (1992). Risk society: Towards a new modernity. Londres: Sage.

Boullier, D. (2009). Les industries de l'attention: au-delà de la fidélisation et de l'opinion. Réseaux, 154.

Boullier, D. (2010). La ville événement. Paris: PUF.

Boullier, D. (2011). Habitèle virtuelle. Revue Urbanisme, 376 (janvier-février), 42-44.

Boullier, D., \& Chevrier, S. (2000). Les sapeurs-pompiers. Des soldats du feu aux techniciens du risque. Paris: PUF. 
Boullier, D., Chevrier S., \& Juguet, S. (2012). Événements et sécurité. Les professionnels de climats urbains. Paris: Presses des Mines.

Bruneteaux, P. (1996). Maintenir l'ordre. Paris: Presses de Science Po.

Chamayou, G. (2013). Théorie du drone. Paris: La fabrique éditions.

Champagne, P. (1984). La manifestation. La production de l'événement politique. Les Actes de la recherche en sciences sociales, 52/53, 19-41.

Chevrier, S., Nogues, T., \& Sauvage, A. (2002). Armées et missions de sécurité intérieure. Les Champs de Mars, 11 (1), 141-152.

Dieu, F. (1993). Gendarmerie et modernité. Paris: Montchrestien.

Dodier, N. (1993). Les appuis conventionnels de l'action. Éléments de pragmatique sociologique. Réseau, 11 (62), 63-85.

Epstein, R., \& Fontaine, A. (2006). Aller en Rave. Un voyage aux marges de la ville. Paris: PUCA Recherche.

Gagnepain, J. (1994). Leçons d'introduction à la théorie de la médiation. LouvainLa-Neuve: Peeters.

Garfinkel, H. (1967). Studies in ethnomethodology. Englewood Cliffs: Prentice Hall.

Goldhaber, M. H. (1997). The attention economy and the Net. First Monday, 2 (4). Consulté le 17 juin 2013, http://firstmonday.org/article/view/519/440

Hall, E. T. (1984). La dimension cachée. Paris: Le Seuil.

Haraway, D. (2003). The companion species manifesto: Dogs, people, and significant otherness. Chicago: Prickly Paradigm Press.

Latour, B. (1992). Nous n'avons jamais été modernes. Essai d'anthropologie symétrique. Paris: La Découverte.

Latour, B. (2006). Changer de société, refaire la sociologie. Paris: La Découverte.

Latour, B. (2012). Enquête sur les modes d'existence: une anthropologie des modernes. Paris: La Découverte.

Latour, B., Jensen, P., Venturini, T., Grauwin, S., \& Boullier, D. (2012). "The whole is always smaller than its parts" - A digital test of Gabriel Tarde's monads. British Journal of Sociology, 63 (4), 590-615.

Lussault, M. (2007). L'bomme spatial. Paris: Seuil.

Mann, P. (1994). Pouvoir politique et maintien de l'ordre. Revue française de sociologie, XXXV-3, 435-455.

Moreau C., \& Sauvage, A. (2006). La fête et les jeunes. Espaces publics incertains. Rennes: Apogée.

Queudrus, S. (2001). La free-party, sociologie d'un vagabondage festif. Quaderni, 44, 129-142.

Sloterdijk, P. (2005). Écumes. Sphères (Tome III). Paris: Libella Maren Sell.

Strauss, A. (1992). La trame de la négociation. Sociologie qualitative et interactionnisme. Paris: L'Harmattan.

Suchman, L. A. (1987). Plans and situated actions: The problem of human-machine communication. Cambridge: Cambridge University Press. 
Viot, P. (2013). Le territoire sécurisé des grandes manifestations contemporaines (Thèse de doctorat). École polytechnique fédérale de Lausanne, Lausanne.

ABSTRACT - Great urban events require the production of safe environments in which participants are able to partake in the event within a protected interior. In this paper we examine three different types of urban events: festivals, demonstrations and sports events. Police forces have become experts in the management of urban climates, relying not on plans but on the creation of conditions in which they themselves and the other actors function. While at the same time using all of their resources in order to maintain some control over the event and not be completely controlled by it.

KEYWORDS - Police, city, event, security, strategy, interior.

RESUMEN - Los grandes eventos urbanos necesitan la producción de ambientes seguros que permitan de vivir el clima del festejo, de la manifestación o de los encuentros deportivos (los terrenos que serán evocados aquí) en un interior protegido. Las fuerzas del orden han adquirido la pericia en el pilotaje de dichos climas, la cual reposa no tanto sobre los planes, sino sobre una aceptación de estar, ellos también, "en el interior" y no por fuera. Las fuerzas del orden movilizan, entonces, todos los recursos del marco establecido y lo completan por propia iniciativa para crear cercos, utilizando también los recursos corporales para mantener despiertos los sentidos ante una situación siempre susceptible de desbordar.

PALABRAS CLAVE - Mantenimiento del orden, ciudad, evento, seguridad, interior. 